\section{Regards sur l'économie allemande}

Bulletin économique du CIRAC

$76 \mid 2006$

Varia

\title{
Ver.di refuse les 40 heures pour construire sa légitimité
}

Isabelle Bourgeois

\section{CpenEdition}

Journals

Édition électronique

URL : http://journals.openedition.org/rea/820

DOI : $10.4000 /$ rea. 820

ISBN : 978-2-8218-0848-5

ISSN : 1965-0787

Éditeur

CIRAC

Édition imprimée

Date de publication : 1 mai 2006

Pagination : 33-36

ISSN : 1156-8992

Référence électronique

Isabelle Bourgeois, "Ver.di refuse les 40 heures pour construire sa légitimité », Regards sur l'économie allemande [En ligne], 76 | mai 2006, document 5, mis en ligne le 19 juin 2008, consulté le 05 mai 2019. URL : http://journals.openedition.org/rea/820 ; DOI : 10.4000/rea.820 


\section{Ver.di refuse les 40 heures pour construire sa légitimité}

\section{Isabelle Bourgeois}

Le syndicat ver.di avait déclenché début février le plus grand mouvement de grève dans les services publics allemands depuis 14 ans. Enjeu: la hausse du temps de travail dans les communes et les Länder. Le passage à 40 heures hebdomadaires, voire plus, se solderait en effet, selon le syndicat, par la suppression de près de 300000 emplois publics. Alors qu'lG Metall mène une politique de sauvegarde de l'emploi grâce à une hausse modularisée du temps de travail conforme aux impératifs de compétitivité de l'industrie (voir REA 68/04 et 75/06), ver.di renoue avec la doctrine du partage du travail. Mais en apparence seulement, car l'argument est instrumentalisé à des fins purement clientélistes. Les actions médiatiques menées par le géant des services visent à construire sa légitimité dans le cadre du processus de modernisation de la fonction publique. Et à consolider sa position face à IG Metall dans le cadre des élections aux Conseils d'établissement (mars) et du renouvellement partiel du directoire du DGB (mai). En développant une fonction tribunicienne, ver.di cherche aussi à s'assurer une certaine représentativité alors qu'un nombre croissant de corporations, dont les médecins des $\mathrm{CHU}$ des Länder, lui tournent le dos.

Les grèves dans la fonction publique ont affecté surtout l'échelon communal : elles trouvent leurs prolongements dans une difficile négociation parallèle au niveau des Länder qui renvoie, elle, à la modernisation des statuts des fonctionnaires fédéraux. Ce jeu de miroirs est la première raison de la virulence des positions défendues par ver.di dans les communes du Bade-Wurtemberg, champ de bataille privilégié puisque s'y déroulaient des élections parlementaires (26 mars) ; celles-ci terminées, les négociations communales ont abouti le 5 avril. A l'échelon des Länder, elles se poursuivaient encore à la fin avril.

La seconde raison réside dans la complexité des statuts de la fonction publique allemande. Les négociations ne concernent pas les fonctionnaires de carrière (Beamte) dont le statut, la rémunération ou la durée du temps de travail sont réglementés par la loi et homogènes sur l'ensemble du territoire. Elles concernent exclusivement les 2 millions d'employés et ouvriers de la fonction et des services publics qui sont soumis, eux, au régime de la négociation collective contractuelle. Cette dernière s'effectue aux trois échelons territoriaux.

Le périmètre de l'emploi public en Allemagne

\begin{tabular}{|lcrrr|}
\hline & Fonctionnaires*) $^{*}$ & Employés & Ouvriers & Total \\
Bund*) & 320000 & 98400 & 74300 & 492700 \\
Länder & 1268400 & 738800 & 108800 & 2116100 \\
Communes & 180000 & 884700 & 327600 & 1392300 \\
Chemins de fer & 52000 & 600 & 2500 & 55000 \\
Administration publique indirecte $\left.{ }^{* *}\right)$ & 64300 & 493800 & 55800 & 613800 \\
Total & $\mathbf{1 8 8 4} \mathbf{4 0 0}$ & $\mathbf{2 ~ 2 1 6 ~ 2 0 0}$ & $\mathbf{5 6 9 0 0 0}$ & $\mathbf{4 6 6 9 \mathbf { 9 0 0 }}$ \\
\hline
\end{tabular}

Source : www.destatis.de; état au 30-06-2004 (actualisation : 03-01-06). *) Beamte; ${ }^{* *}$ ) dont militaires (186 700); $\left.{ }^{* * *}\right)$ Agence fédérale pour l'emploi, Bundesbank, organismes de sécurité sociale, etc.

Ne sont concernés actuellement que les deux Länder et les communes. Au niveau des Länder, les partenaires sociaux en présence sont: la Tarifgemeinschaft deutscher Länder ( $T d L)$, fédération des Länder en tant qu'employeurs ; en face, ver.di, membre de la Confédération syndicale DGB, et la $D B B$
Des négociations complexes...

... pour une fonction publique elle aussi complexe 
Enjeu des négociations : la durée du temps de travail
Tarifunion (350 000 adhérents) qui est affiliée au syndicat des Beamte, le Deutscher Beamtenbund (DBB). A l'échelon des communes, ces derniers affrontent les fédérations des employeurs communaux: les Kommunale Arbeitgeberverbände (KAV), constituées au sein de chaque Land.

A la différence des négociations qui ont débuté au même moment dans la métallurgie (voir REA 75/06), celles qui touchent la fonction publique ne portent pas sur des questions salariales. Les grèves, démarrées le 6 février dans les communes du Bade-Wurtemberg, portaient sur la durée du temps de travail. Alors que les KAV souhaitaient introduire les 40 heures, ver.di défendait le statu quo: 38,5 heures. Les communes du Bade-Wurtemberg avaient été les premières à mettre en œuvre une disposition contenue dans l'accord relatif aux salariés de la fonction publique communale et fédérale (Tarifvertrag öffentlicher Dienst, TVöD) signé le 13-09-2005. II s'agit d'une clause d'ouverture qui permet aux communes de négocier une hausse du temps de travail.

\section{Le Tarifvertrag öffentlicher Dienst (TVöD) du 13 septembre 2005}

Cet accord a été longuement négocié. En janvier 2003, les partenaires sociaux concernés au niveau du Bund et des communes s'étaient mis d'accord sur une réforme en profondeur du régime en vigueur pour les salariés contractuels de la fonction publique (" accord de Potsdam », voir REA 69/04). Les négociations ont abouti en septembre 2005. Le TVöD a été signé, au niveau patronal, par le Bund et l'Union des KAV; au niveau syndical, par la DBB Tarifunion et ver.di. II est entré en vigueur le $1^{\text {er }}$ octobre 2005. Ce nouveau texte modernise le régime des salariés du Bund et des communes, de même que celui de quelque 8 millions de salariés assimilés (notamment dans les universités), soumis jusque là au Bundes-Angestelltentarifvertrag (BAT). Cet accord, dont les dispositions prendront effet progressivement jusqu'en 2007, comporte 4 volets :

- une harmonisation des régimes : le BAT est supprimé et remplacé par le TVöD, convention désormais unique ;

- une harmonisation des statuts : la distinction entre employés et ouvriers est supprimée :

- un changement de paradigme dans la gestion des carrières et des émoluments : les critères liés à l'ancienneté et au statut sont remplacés par des critères de performance débouchant sur une fixation des salaires au mérite et un dispositif de primes d'efficience ; la grille des salaires est simplifiée et ne comporte plus que 15 échelons ;

- une modularisation du temps de travail : sur la base d'une durée hebdomadaire moyenne de 39 heures à l'est comme à l'ouest, des dérogations peuvent être négociées (corridors ; maximum : 45 heures).

Les Länder avaient refusé de participer à l'élaboration du TVöD ; à leur échelon, les négociations se poursuivent donc. Mais ils sont divisés notamment sur la durée du temps de travail. Plus généralement, leurs réticences s'expliquent par la question du transfert, du Bund vers les Länder, des compétences relatives au régime des fonctionnaires stricto sensu; elle est au cœur des travaux sur la réforme du fédéralisme qui doivent aboutir d'ici l'été. Cette question se double de la réforme en cours des statuts des Beamte. Après que le ministère fédéral de l'Intérieur (employeur), le syndicat des fonctionnaires DBB Beamtenbund, la DBB Tarifunion et ver.di étaient parvenus, le 4 octobre 2004, à un accord sur les lignes directrices de la réforme (très proches de celles du TVöD), un projet de loi avait été formulé le 15 juin 2005 . Il doit être définitivement adopté en conseil des ministres le 25 mai 2006 avent d'être soumis au vote parlementaire. Son entrée en vigueur est prévue le $1^{\mathrm{er}}$ juin 2006.

Les accords conclus à Hambourg, en Basse-Saxe et dans le Bade-Wurtemberg

Temps de travail : un cheval de bataille pour ver.di...
A Hambourg et en Basse-Saxe, deux accords avaient été conclus respectivement le $1^{\text {er }}$ et le 15 mars. Le second est un compromis classique : pour tous les salariés, le temps de travail est maintenu à 39 heures hebdomadaires ; mais pour les employés des caisses d'épargne et de l'administration s'y ajoutent deux journées annuelles de travail ; les autres catégories d'agents sont tenues de consacrer entre 1 et 3 journées, non rétribuées, à la formation continue. L'accord de Hambourg est très controversé : s'il introduit un corridor compris entre 38 et 40 heures, la durée du temps de travail est échelonnée selon l'âge et la catégorie indiciaire du salarié, ce qui revient à réintroduire par la bande des critères de statut et d'ancienneté, pourtant abolis par le TVöD. Dans le Bade-Wurtemberg, l'accord du 5 avril porte le temps de travail à 39 heures.

Dans les négociations, le syndicat ver.di (suivi par la DBB Tarifunion), a enfourché un de ses grands chevaux de bataille : la question du temps de travail, présentée sous l'angle de la conciliation entre vie familiale et professionnelle. Cette revendication récurrente a pour principale fonction de créer un élan fédérateur au sein d'un conglomérat en quête de cohésion interne (Pautrat, 2005) ; elle se traduit par une politique conventionnelle 'qualitative', c'est-à-dire portant sur des grandes questions de société, et non plus strictement salariale ou relative à l'organisation du travail - impossible au demeurant, tant les services sont protéiformes. La fonction publique semble en outre plus réceptive à ce type de revendication que les employés du secteur privé des services. Dans le commerce de détail à Berlin, ver.di avait conclu au début de l'année une convention portant 
sur une flexibilisation du temps de travail via le régime de dérogation aux minima de branche (donc une hausse selon le modèle d'IG Metall) avec, pour contrepartie, une augmentation modérée des salaires $(1 \%)$. Cet exemple révèle le positionnement ambigu d'un géant des services qui fédère quelque 1000 métiers et représente les intérêts contradictoires des salariés du public et du privé.

Le temps de travail dans les communes offre une opportunité rêvée pour lancer dans l'espace public un débat destiné à entretenir l'image du syndicat. Mais elle comporte un risque majeur : l'opinion considère comme légitime la quête d'efficience des employeurs communaux demandant à des salariés qui ont la sécurité de l'emploi de travailler 18 minutes de plus par jour. De surcroît, dans cette 'petite Suisse' qu'est le Bade-Wurtemberg (voir REA 74/05), une grève des éboueurs est du plus mauvais effet. Les citoyens, qui y ont une part active dans la politique communale, approuvent un management public à la recherche lui aussi de compétitivité. Ils sont conscients aussi, plus généralement, de l'énorme endettement des communes allemandes (plus de 100 milliards $€$ à la fin 2005 ; Destatis) dont les dépenses sociales sont en nette augmentation notamment depuis l'entrée en vigueur de la loi Hartz IV (+11\% en 2005 pour un total de 35,5 milliards $€$ ). Elles sont amenées à réduire leurs investissements, tombés à 33 milliards $€$ et à contenir leur masse salariale (41 milliards $€$ ). Sentant tourner le vent, ver.di avait donc changé de tactique à la fin mars, prenant pour cible les employeurs et non plus les usagers.

Si ver.di se crispe tant sur son cheval de bataille, c'est parce que le conflit communal en cache d'autres. D'abord, la libéralisation d'une part croissante des services publics. Elle force ver.di à multiplier les accords catégoriels pour éviter la désyndicalisation et la constitution hors de son champ de syndicats corporatistes (dernier exemple en date : les 146000 médecins des CHU des Länder). La lutte contre le « dumping salarial » dans les services publics où, de plus en plus, coexistent régimes public et privé, est un des principaux axes stratégiques de ver.di depuis plusieurs années ; elle n'est certes pas l'objet des négociations actuelles, mais interfère en toile de fond dans la mesure où elle pose la question de la représentativité dans un secteur régi par d'innombrables accords conventionnels. Elle interfère également dans la mesure où elle nourrit un autre cheval de bataille de ver.di : le revendication d'un salaire minimum légal.

S'y ajoute, toujours en toile de fond, un autre encore : son opposition farouche à la privatisation ou le démantèlement des entreprises communales (Stadtwerke) qui gèrent les transports et assurent l'approvisionnement en eau, gaz, électricité ou chauffage. Ce chantier, ancien lui aussi, est stratégique pour un syndicat qui voit fondre sa clientèle ( $80 \%$ des éboueurs par exemple sont dans le privé) et ses adhérents (il en a perdu un demi-million l'an dernier), et qui cherche à préserver la prépondérance de l'ancien syndicat du secteur public et des transports, ÖTV, aujourd'hui fondu dans ver.di (2,36 millions d'adhérents). C'est de loin la formation la plus importante en son sein : ÖTV avait 1,5 million de membres en 2000. C'est aussi d'ÖTV qu'est issu Frank Bsirske, le président du directoire du géant des services. Cet expert en management public cumule les pouvoirs stratégiques. Pour n'en citer que les principaux : définition de la stratégie du syndicat, audit interne, affaires européennes, relations internationales, communication interne et externe. La cohésion de ver.di « tient plus aux personnes qu'aux fonctions », avoue-t-on de source syndicale.

La personnalité du communicateur Frank Bsirske (membre des Verts) joue un rôle clef dans le conflit communal. Car celui-ci lui permet d'en nourrir un autre, qui se déroule en parallèle, et où ver.di est en position de faiblesse (une grève des universitaires n'a qu'une faible visibilité) : la difficile négociation d'un accord sur la fonction publique des Länder. L'an dernier, la fédération des employeurs TdL avait dénoncé la convention sur le temps de travail ; Bavière, Basse-Saxe, Bade-Wurtemberg, Hesse et Rhénanie du Nord-Westphalie avaient procédé à une hausse (42 heures en Bavière et en Hesse) pour les fonctionnaires et sa-
... et prétexte à une campagne d'image

Une campagne stratégique pour revendiquer un SMIC légal...

... et pour rester influent dans les services publics

Les négociations sont ouvertes dans les Länder aussi 
Par-delà se joue la réforme du statut des fonctionnaires lariés nouvellement recrutés. Les impératifs des Länder sont les mêmes que ceux des communes : accroître l'efficience de l'administration (en en modernisant aussi la GRH) tout en contenant une masse salariale qui représente $40 \%$ de leurs dépenses. Ils privatisent à tout va. Depuis le début des négociations sur une révision des régimes de la fonction publique, ver.di s'oppose farouchement aux 40 heures. Or les Länder campent sur leurs positions (d'autant que le gouvernement fédéral a passé la durée du temps de travail des Beamte à 41 heures). Et s'ils se déclarent prêts à adopter eux aussi le $T V o ̈ D$, ils veulent en outre harmoniser le régime des fonctionnaires avec celui des contractuels.

Mais les Länder sont divisés. Deux lignes de conflit les opposent au sein de la TdL. La première oppose les avocats d'une hausse, qui se recrutent dans les Länder à gouvernement majoritairement CDU/CSU, et les 5 Länder SPD, qui soutiennent ver.di pour des raisons idéologiques. La seconde traverse Bund et Länder. Le gouvernement fédéral s'apprête à adopter son projet de loi sur la réforme du statut des Beamte : il serait uniforme sur l'ensemble du territoire. Or dans le cadre de la réforme du fédéralisme, les compétences pour le statut et la rémunération des Beamte devraient être transférée aux Länder, ce qui est logique puisque, dans le partage fonctionnel des compétences entre Bund et Länder, c'est à ces derniers que revient le pouvoir administratif (celui du Bund est résiduel). II s'agit donc aussi de trouver une meilleure articulation entre ces deux réformes et le $T V O ̈ D$. Les Länder qui ont déjà haussé le temps de travail se réjouissent à la perspective de ce complément de souveraineté qui leur permettra de moderniser leur administration. Le nouveau gouvernement du BadeWurtemberg se propose dans ce contexte à relever à 67 ans l'âge légal de départ à la retraite de ses Beamte. Ver.di s'oppose farouchement à une diversification des régimes qui réduirait mécaniquement son influence.

« Normalement, ON NE fait PAS GRÈVE PENDANT LES NÉgociations », s'insurge Hartmut Möllring, ministre des Finances de Basse-Saxe et président de la TdL. Et de fait, ver.di déroge à toutes les règles du partenariat social allemand. Sa création en 2001 cherche à apporter une réponse institutionnelle aux mutations des activités ; elle est défensive. Culturellement, ver.di est en rupture avec un syndicalisme assumant ses responsabilités dans le délicat équilibre d'intérêts entre capital et travail, à l'image d'IG Metall (2,38 millions d'adhérents). Sa tactique en est le reflet: dès avant d'accéder à la tête de ver.di, Frank Bsirske la décrivait ainsi : "plus Greenpeace que puissance gréviste ». Ses finances ne lui permettant pas d'engager une grève d'envergure, il multiplie les actions ponctuelles, cherchant l'épreuve de force dans l'espace public. En cela, il s'adapte aussi à la médiatisation de la société allemande qui requiert des actions spectaculaires pour occuper l'agenda. Cela le rapproche d'ATTAC, son partenaire privilégié sur la Directive 'Services' (voir REA 71/05). Pour le renouvellement partiel du directoire du DGB en mai, deux candidats sont en lice : un membre d'IG Metall (SPD) et une représentante de ver.di, membre du parti des Verts et proche d'ATTAC. Si ver.di campe sur ses positions dans les négociations sur le service public, c'est moins pour y défendre l'emploi et les conditions de travail que pour renforcer sa position face à IG Metall et pour gagner sa légitimité dans le partenariat social allemand. Mais en cultivant une action en rupture avec la culture syndicale allemande, ver.di joue son avenir.

\section{Indications bibliographiques :}

PaUtRat M.-H., "Ver.di : un géant sans cohésion », in BouRgeols I. (dir.), Le modèle social allemand en mutation, CIRAC, 2005

RENNEBERG P., "Veränderte Arbeitsbeziehungen - Veränderung der Arbeitskampfformen ? ", WSI-Mitteilungen, 5/2005

Wessels B. "Gewerkschaften in der Mediengesellschaft », in Schroeder W., Wessels B. (eds), Die Gewerkschaften in Politik und Gesellschaft der Bundesrepublik Deutschland, Wiesbaden, 2003

www.verdi.de. 